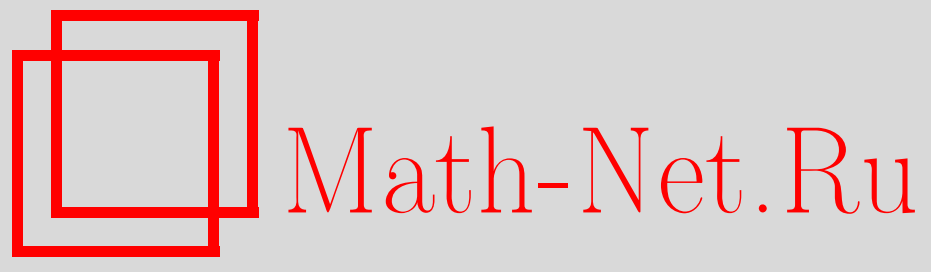

Г. Г. Ильюта, Соотношения для многочлена Александеpa, УMH, 2008, том 63, выпуск 3, 161-162

DOI: https://doi.org/10.4213/rm9181

Использование Общероссийского математического портала Math-Net.Ru подразумевает, что вы прочитали и согласны с пользовательским соглашением http://www . mathnet.ru/rus/agreement

Параметры загрузки:

IP: 54.198 .67 .100

26 апреля 2023 г., 12:49:45

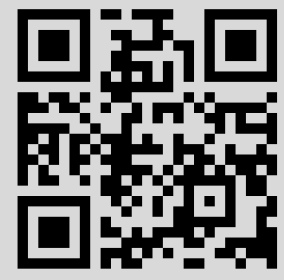




\section{Соотношения для многочлена Александера}

\section{Г. Г. Ильюта}

Один из подходов к построению инвариантов узлов состоит в использовании соотношений, связывающих значения инварианта для некоторого набора перестроек узла. Например, для любого квантового инварианта имеется соотношение, определяемое минимальным многочленом $R$-матрицы [1], в частности, имеем соотношение Конвея для многочлена Александера. Мы обобщим соотношение Конвея в другом направлении, а именно, докажем существование линейных соотношений для многочленов Александера, отвечающих широкому классу наборов перестроек (единственное требование к этим наборам состоит в том, что они должны быть достаточно большими). Доказательство конструктивно - вычисление коэффициентов в соотношении для данного набора перестроек сводится к нахождению линейной зависимости в легко вычисляемом наборе векторов. Тот же подход позволяет найти соотношения для характеристических многочленов операторов из группы монодромии особенности. Ключевыми средствами для доказательства являются формула для многочлена Александера $A_{b}(q)$ замыкания косы $b$ из группы кос $B_{m}(r-$ представление Бурау) [2]: $A_{b}(q)=\operatorname{det}(\operatorname{Id}-r(b)) /\left(q^{m-1}+\cdots+1\right)$; и формула для блочного определителя, использовавшаяся уже в теории многочленов Александера узлов (построение матрицы представления для модуля гомологий циклического накрытия дополнения к узлу) [3], [4] и особенностей (формула для дзета-функций монодромии серии особенностей) [5], [6]: $\operatorname{det}\left(\mathrm{Id}-h_{1} \cdots h_{k}\right)=\operatorname{det}(\mathrm{Id}-H)$, где $H-$ блочная матрица с элементами $H_{i i+1}=h_{i}$ для $i<k, H_{k 1}=h_{k}$, остальные элементы равны 0. Полученные соотношения аналогично [7] приводят к соотношениям для обобщенных разделенных разностей, определенных по многочлену Александера. Мы приведем соответствующие соотношения для обобщенного интерполяционного многочлена Лагранжа.

Инварианту кос и любой косе можно сопоставить бесконечный вектор значений инварианта на произведениях этой косы на все косы. Набору кос соответствует конфигурация векторов в порожденном этими векторами пространстве (конфигурация гиперплоскостей в двойственном пространстве). В [8] определены пространства высших соотношений для конфигурации гиперплоскостей. Конфигурация называется формальной, если первое пространство соотношений порождается соотношениями длины (количество слагаемых) 3. Таким образом, если соотношения Конвея порождают все соотношения, то мы приходим к формальной конфигурации гиперплоскостей.

Рассмотрим последовательность $k$-элементных наборов кос $G=\left(g_{1}, \ldots, g_{k}\right), F_{i}=$ $\left(f_{1 i}, \ldots, f_{k i}\right), i=1,2, \ldots$ и обозначим через $v_{i}$ косу $f_{1 i} g_{1} \cdots f_{k i} g_{k}$. Предположим, что для любого фиксированного $j$ все матрицы $r\left(f_{j i}\right)$ могут отличаться от единичной в одних и тех же $p_{j}$ строках и $q_{j}$ столбцах (возможно, различных для разных $j$ ). Пусть $p=p_{1}+\cdots+p_{k}, q=q_{1}+\cdots+q_{k}$ и $s=C_{p+q}^{p}$.

Предложение 1. Между многочленами Александера $A_{v_{1}}(q), \ldots, A_{v_{s+1}}(q)$ существует нетривиальная линейная зависимость, коэфбициенты которой не зависят om $G$.

ДокАЗАТЕЛЬСтво. Применяем к $r\left(v_{1}\right)$ формулу для блочного определителя. В правой части этой формулы к каждой нечетной блочной строке прибавляем следующую и раскладываем полученный определитель по блочным столбцам, отвечающим $F_{1}$. Вектор максимальных миноров блочных столбцов, отвечающих $F_{1}$, содержит не более чем $s$ ненулевых элементов и поэтому $s+1$ подобных векторов линейно зависимы.

Работа поддержана грантами РФФИ-07-01-00593, INTAS-05-7805 и НШ-4719.2006.1. 
Если функция $A(X, Y)$ допускает разделение переменных вида $A(X, Y)=C_{1}(X) \times$ $D_{1}(Y)+\cdots+C_{l}(X) D_{l}(Y)$ (в предыдущем доказательстве разделены переменные $F$ и $G)$, то аналогично можно получить соотношение для конечной матрицы $\left(A\left(X_{i}, Y_{j}\right)\right)=\left(C_{k}\left(X_{i}\right)\right)\left(D_{k}\left(Y_{j}\right)\right)^{t}$ при различных значениях $X_{i}$. Разделение переменных сохранится, если взять образы матриц при каком-либо сохраняющем произведение представлении.

Для множества $S$ и поля $F$ рассмотрим функцию $d: S^{2} \rightarrow F$, равную 0 при равных аргументах, например, $d(f, g)=A_{f g^{-1}}(q)$. Пусть $Z=\left\{z_{1}, z_{2}, \ldots\right\} \subset S$, $Z_{n}=\left\{z_{1}, \ldots, z_{n}\right\}, d\left(z_{i}, z_{j}\right) \neq 0$ при $i \neq j$. Для функции $f$ на $S$ определим разделенные разности, интерполяционный многочлен Лагранжа и ряд Ньютона формулами

$$
\begin{aligned}
D_{Z_{n}}[f] & =\sum_{i=1}^{n} f\left(z_{i}\right) / \prod_{j \neq i} d\left(z_{i}, z_{j}\right), \\
I_{Z_{n}}[f] & =\sum_{i=1}^{n} f\left(z_{i}\right) \prod_{j \neq i} d\left(z, z_{j}\right) / d\left(z_{i}, z_{j}\right), \\
N_{Z}[f] & =\sum_{i \geqslant 0} D_{Z_{i+1}}[f] \prod_{j=1}^{i} d\left(z, z_{j}\right) .
\end{aligned}
$$

ЛЕмма 1. Для $i=1, \ldots, n$ справедлива формула

$$
I_{Z_{n}}\left[d\left(z, z_{i}\right) f(z)\right]=d\left(z, z_{i}\right) I_{Z_{n} \backslash z_{i}}[f(z)] .
$$

СлеДСтвиЕ 1. Если функиия $d$ удовлетворяет соотношению $c_{1} d\left(z, z_{1}\right)+\cdots+$ $c_{k} d\left(z, z_{k}\right)$, mо для $k \leqslant n$

$$
\sum_{i=1}^{k} c_{i} d\left(z, z_{i}\right) I_{Z_{n} \backslash z_{i}}[f(z)]=0 .
$$

Следствие 2 (правило Лейбница). Для функиий $f$ u $h$ на $S$ справедлива формула

$$
I_{Z_{n}}\left[N_{Z}[f] h\right]=\sum_{i=0}^{n-1} D_{Z_{i+1}}[f] I_{Z_{n} \backslash Z_{i}}[h] \prod_{j=1}^{i} d\left(z, z_{j}\right) .
$$

\section{Список литературы}

[1] V. G. Turaev, Invent. Math., 92:3 (1988), 527-553. [2] J.S. Birman, Braids, links, and mapping class groups, Ann. Math. Studies, 82, Princeton Univ. Press, Princeton, NJ; Univ. Tokyo Press, Tokyo, 1974. [3] H. Seifert, Abh. Math. Sem. Univ. Hamburg, 11 (1935), 84-101. [4] S. Ocken, Proc. Amer. Math. Soc., 110:4 (1990), 1063-1067. [5] D. Siersma, Comment. Math. Helv., 65:2 (1990), 181-197. [6] A. Némethi, J. Algebraic Geom., 2:1 (1993), 1-23. [7] Г. Г. Ильюта, УМH, 61:4 (2006), 189-190; англ. пер.: G. G. Il'yuta, Russian Math. Surveys, 61:4 (2006), 775-777. [8] K. A. Brandt, H. Terao, Discrete Comput. Geom., 12:1 (1994), 49-63.

\section{Г. Г. Ильюта (G. G. Ilyuta)}

Московский государственный открытый педагогический университет им. М. А. Шолохова

E-mail: ilyuta@mccme.ru
Представлено С. М. Гусейн-Заде Принято редколлегией 04.02 .2008 